\title{
ON THE DETERMINATION OF A HILL'S EQUATION FROM ITS SPECTRUM
}

\author{
BY WALLACE GOLDBERG ${ }^{1}$
}

Communicated by Cathleen Morawetz, March 26, 1974

A Hill's equation is an equation of the form:

$$
y^{\prime \prime}+[\lambda-q(z)] y=0, \quad q(z+\pi)=q(z),
$$

where $q(z)$ is assumed to be integrable over $[0, \pi]$. Without loss of generality, it is customary to assume that $\int_{0}^{\pi} q(z) d z=0$. The discriminant of (1) is defined by

$$
\Delta(\lambda)=y_{1}(\pi)+y_{2}^{\prime}(\pi),
$$

where $y_{1}$ and $y_{2}$ are solutions of (1) satisfying $y_{1}(0)=y_{2}^{\prime}(0)=1$ and $y_{1}^{\prime}(0)=$ $y_{2}(0)=0$.

The set of values of $\lambda$ for which $|\Delta|>2$ consists of a finite or an infinite number of finite disjoint intervals and one infinite interval. These intervals are called instability intervals, since (1) has no solution which is bounded for all real $z$ in these intervals. When $|\Delta|<2$, all solutions of (1) are bounded for all real $z$ and the corresponding intervals are called stability intervals. Pertinent information about stability and instability intervals of (1) can be found in Magnus and Winkler [1].

The following result has been proved:

THEOREM. If $q(z)$ is real and integrable, and if precisely $n$ finite instability intervals fail to vanish, then $q(z)$ must satisfy a differential equation of the form

$$
q^{(2 n)}+H\left(q, q^{\prime}, \cdots, q^{(2 n-2)}\right)=0, \text { a.e. }
$$

where $H$ is a polynomial of maximal degree $n+2$.

Borg [2], Hochstadt [3] and Ungar [4] proved this theorem for the case $n=0$, i.e. when all finite instability intervals vanish, and found that

$$
q(z)=0 \text {, a.e. }
$$

For the case $n=1$, Hochstadt [3] showed that $q(z)$ is the elliptic function which satisfies

$$
q^{\prime \prime}=3 q^{2}+A q+B, \text { a.e. }
$$

AMS (MOS) subject classifications (1970). Primary 34B30; Secondary 34E05.

${ }^{1} \mathrm{I}$ thank my advisor, Harry Hochstadt, for his generous advice and guidance. 
where $A$ and $B$ are constants. (3) and (4) are equivalent to (2) for the cases $n=0$ and 1 , respectively. In particular, for the case $n=2$, the explicit expression of (2) is

$$
q^{(4)}=10 q q^{\prime \prime}+A q^{\prime \prime}+5\left(q^{\prime}\right)^{2}-10 q^{3}+B q^{2}+C q+D, \text { a.e. }
$$

where $A, B, C$ and $D$ are constants.

Erdélyi [5] investigated a Hill's equation where $q(z)$ is a Lamé function and discovered situations where all but a finite number of finite instability intervals vanish. (4) provides a converse to some of his results.

Lax [6], through the study of partial differential operators, derived sufficient conditions for the vanishing of all but $n$ finite instability intervals. These conditions coincide with (3), (4) and (5) for the cases $n=0,1$ and 2 , respectively. Whether there exist equivalent necessary conditions for higher values of $n$ is still an open question.

The proof of the Theorem is accomplished by investigating the related problem

$$
u^{\prime \prime}+[\lambda-q(z+\tau)] u=0 ; \tau \text { real, arbitrary }
$$

and by assuming the result [3] that $q(z)$ is infinitely differentiable a.e. when $n$ finite instability intervals fail to vanish.

In [3], Hochstadt showed that (6), when subject to $u(0)=u(\pi)$, has eigenvalues $\mu_{n}(\tau)$, where $\mu_{i}(\tau)$ lies in the $i$ th finite instability interval of (1). Furthermore, when precisely $n$ finite instability intervals fail to vanish

$$
u_{2}(\pi) \prod_{i=1}^{n}\left[\lambda-\mu_{i}(0)\right]=y_{2}(\pi) \prod_{i=1}^{n}\left[\lambda-\mu_{i}(\tau)\right]
$$

where $u_{2}(t)$ denotes the solution of (6) which satisfies $u_{2}(0)=0$ and $u_{2}^{\prime}(0)=1$. Suitable asymptotic expressions of $u_{2}(\pi)$ and $y_{2}(\pi)$ have been developed and our result follows upon their substitution into this equation. The details will appear in a later paper.

\section{REFERENCES}

1. W. Magnus and S. Winkler, Hill's equation, Interscience Tracts in Pure and Appl. Math., no. 20, Interscience, New York, 1966. MR 33 \#5991.

2. G. Borg, Eine Umkehrung der Sturm-Liouvilleschen Eigenwertaufgabe. Bestimmung der Differentialgleichung durch die Eigenwerte, Acta Math. 78 (1946), 1-96. MR 7, 382.

3. H. Hochstadt, On the determination of a Hill's equation from its spectrum, Arch. Rational Mech. Anal. 19 (1965), 353-362. MR 31 \#6019.

4. P. Ungar, Stable Hill equations, Comm. Pure Appl. Math. 14 (1961), 707-710. MR 31 \#423.

5. A. Erdélyi et al., Higher transcendental functions. Vol. III, McGraw-Hill, New York, 1955, p. 63ff. MR 16, 586.

6. P. Lax, Personal communication.

Department of Mathematics, Queens College, Flushing, New York 11367 